\title{
Asistencia pedagógica virtual y desempeño docente en tiempos de pandemia en la I.E. Pedro pablo Atusparia de Nuevo Chimbote, 2021
}

\author{
Javier Eduardo Barreno Flores \\ Jbarrenof15@ucvvirtual.edu.pe \\ https://orcid.org/0000-0001-7572-0190 \\ Universidad César Vallejo \\ Nuevo Chimbote - Perú
}

\section{RESUMEN}

Esta investigación centrada en los docentes, en tiempos de la pandemia originada por la COVID19, tuvo como propósito demostrar que la asistencia pedagógica virtual mejora significativamente el desempeño docente en la I.E. N 88227 "Pedro Pablo Atusparia" de Nuevo Chimbote, 2021. Fue un estudio aplicado de tipo experimental con diseño pre experimental, con pretest y postest, para lo cual se aplicó una encuesta virtual a fin de recoger información sobre el efecto de la variable independiente en una muestra de 31 docentes durante un mes y medio. Estos instrumentos validados por expertos y con una confiabilidad de 0,940 según la prueba Alfa de Cronbach, lograron determinar que el Programa de Asistencia Pedagógica Virtual mejoró significativamente el desempeño docente en tiempos de pandemia del docente atuspariano pues para el análisis de la hipótesis se aplicó la prueba T de Student y se logró obtener un valor T observado de 8,612 considerado como superior al valor T tabular 1,697 (30 grados de libertad). Además, se concluyó en que el programa mejoró significativamente la tarea de planificación del docente, así como su capacidad de fomentar una buena convivencia virtual, sus estrategias didácticas y sus estrategias de evaluación en el trabajo a distancia.

Palabras clave: asistencia pedagógica; virtualidad; desempeño docente; tecnología; aprendizaje social. 


\title{
Virtual pedagogical assistance and teaching performance in times of pandemic in the I.E. Pedro pablo Atusparia from Nuevo Chimbote, 2021
}

\begin{abstract}
This research focused on teachers, in times of the pandemic caused by COVID19, had the purpose of demonstrating that virtual pedagogical assistance significantly improves teaching performance in the I.E. $\mathrm{N}$ ○ 88227 "Pedro Pablo Atusparia" from Nuevo Chimbote, 2021. It was an applied experimental study with a pre-experimental design, with pretest and posttest, for which a virtual survey was applied in order to collect information on the effect of the independent variable in a sample of 31 teachers for a month and a half. These instruments, validated by experts and with a reliability of 0.940 according to the Cronbach's Alpha test, were able to determine that the Virtual Pedagogical Assistance Program significantly improved the teaching performance in times of pandemic of the Atusparian teacher, since the test was applied for the analysis of the hypothesis Student's T value and an observed T value of 8.612 was obtained, considered to be higher than the tabular $\mathrm{T}$ value of 1.697 (30 degrees of freedom). In addition, it was concluded that the program significantly improved the planning task of the teacher, as well as their ability to promote a good virtual coexistence, their didactic strategies and their evaluation strategies in distance work.
\end{abstract}

Keywords: pedagogical assistance; virtuality; teaching performance; technology; social learning.

Artículo recibido: 20 diciembre. 2021 Aceptado para publicación: 10 enero 2022 Correspondencia: Jbarrenof15@ucvvirtual.edu.pe Conflictos de Interés: Ninguna que declarar 


\section{INTRODUCCIÓN}

El año 2020 significó para casi todas las naciones del planeta el sometimiento de nuestras grandes libertades personales y de nuestras facultades sociales ante la mortal presencia de un virus denominado COVID-19, el cual emergió tan letal y vertiginosamente que, en poco tiempo, los países de los cinco continentes cerraban sus fronteras y ordenaban a sus pobladores a guardar cuarentena; y, desde que este aislamiento social empezó, en casi todos los países del orbe, los centros educativos públicos o privados y los centros de nivel superior (Santiago et al., 2020), ante semejante ola de contagios y de muerte masiva, no tuvieron mejor opción que cerrar sus puertas para reducir el contagio sobre todo en los niños y adolescentes.

En medio de esta pandemia, el ingreso al mundo virtual mediante el uso de dispositivos electrónicos se constituyó en una demanda muy importante para salvar el proceso educativo en nuestro país (Rojas y Cachay, 2021). Sin embargo, quizá el problema más agudo relacionado con el aprendizaje de los estudiantes fue descubrir la limitada capacidad de enseñanza virtual que han tenido los profesores a pesar de muchos programas de actualización antes de la pandemia (Vásquez y Henríquez, 2016; VarelaOrdorica y Valenzuela-González, 2020). Ha sido una realidad largamente arraigada donde las TIC no eran consideradas imprescindibles y ahora resulta que, por el contrario, se han convertido en un prerrequisito para el trabajo pedagógico, eficiente y eficaz (Revilla-Mendoza \& Palacios-Jimenez, 2020).

Inevitablemente, surgen las preguntas: ¿Habrán estado los docentes a la altura de las circunstancias al asumir el nuevo reto de una educación a distancia y/o virtual? ¿Cómo atender a todos los estudiantes sabiendo que no todos tienen la misma conexión? ¿Tendrán los docentes problemas de conectividad? ¿Estarán en condiciones de planificar, evaluar y reflexionar sobre su práctica pedagógica?

Desde el 6 de abril del mismo año, los docentes peruanos iniciaron una nueva etapa muy significativa en su vida laboral: Ser mediadores del aprendizaje utilizando medios virtuales y herramientas tecnológicas a través de una plataforma implementada por el MINEDU denominada "Aprendo en casa".

La DRE Ancash tuvo una significativa participación en el intento de reorganizar las fuerzas magisteriales de la región en favor del aprendizaje de los niños y adolescentes, brindando a los docentes lineamientos y orientaciones sobre cómo desplegar nuestro 
trabajo pedagógico y la evaluación formativa en forma virtual y de la mejor manera posible. Un escenario donde la capacidad del docente era clave para el logro de los estándares de nuestro CNEB.

En medio de la pandemia, surgió la necesidad de un trabajo colegiado virtual donde la asistencia pedagógica se constituya en uno de los ejes claves para el trabajo efectivo del profesor y el aprendizaje eficaz de los discentes. Era palpable la necesidad de los docentes de una preparación virtual intensiva para que no decaiga más su importante desempeño. Hay una gran necesidad de manejar las herramientas tecnológicas de planificación, organización, seguimiento y evaluación, todo a nivel virtual, para un proceso educativo de acuerdo a los propósitos trazados en estos tiempos de pandemia (Huamani et al., 2019; Fardoun et al., 2020; Tamay-Chimborazo et al., 2020).

Por eso, en esta realidad problemática descrita, se plantea como propuesta: la Asistencia Pedagógica Virtual (APEVI), la cual se erige como una alternativa que pueda hacer posible la mejora del trabajo de los maestros en este nuevo escenario donde niños, adolescentes, jóvenes y adultos despliegan sus esfuerzos por una educación de buena calidad. Las herramientas TIC deben ser consideradas como elementos educativos significativos en la educación en tiempos de pandemia (Sianes y Sánchez, 2020).

Por eso, el objetivo general de esta investigación pretendió demostrar cómo la Asistencia Pedagógica Virtual mejoraría el desempeño docente en tiempos de pandemia en la I.E. Pedro Pablo Atusparia de Nuevo Chimbote y sus principales objetivos específicos fueron demostrar el mismo mejoramiento en aspectos clave de dicho desempeño como la planificación colegiada, la convivencia virtual, el adiestramiento en estrategias didácticas virtuales y en estrategias de evaluación formativa por lo cual, la Hi se expresó como que "la asistencia pedagógica virtual mejora significativamente el desempeño docente en tiempos de pandemia en la I.E. PPA de Nuevo Chimbote y la Ho se expresó así: "La asistencia pedagógica virtual no mejora el desempeño docente en tiempos de pandemia en la I.E. PPA de Nuevo Chimbote".

Este estudio se sustentó epistemológicamente en el trabajo de eminentes psicólogos y pedagogos cuyas teorías universalmente aceptadas, contribuyen a la investigación sobre la importancia de la asistencia pedagógica virtual para mejorar el desempeño docente especialmente en estos tiempos de pandemia a causa del virus COVIDSARS-2 (Santiago et al., 2020; Aramayo et al., 2020; Gerbaldo y Granato, 2020). 
La variable denominada Asistencia Pedagógica Virtual se basa, en primer lugar, en la Teoría del Aprendizaje Social de Albert Bandura quien sostiene que el individuo necesariamente aprende de la interacción con otras personas al observar y repetir las acciones y actitudes realizadas por aquellas (Bandura, 1975). Así mismo, Carl Rogers, citado por Schultz y Schultz, sostenía que cualquiera que sea el caso de encuentro entre personas y, especialmente si forma parte del trabajo como educadores, no se debe perder de vista que la calidad de dicha interacción es el quid más importante para lograr las metas trazadas. La relación personal definirá siempre el desarrollo de la personalidad del ser humano (Schultz y Schultz, 2010).

Nuestro ejercicio pedagógico vuelve a nacer, pero ahora con el compromiso de buscar el reencuentro entre todos los agentes educativos con el soporte emocional necesario. El acompañamiento es la estrategia principal para pensar en un éxito educativo cuando termine el aislamiento obligatorio (Espinosa-Izquierdo et al., 2021; Gerbaldo y Granato, 2020). Es una estrategia que guarda la intención de elevar la calidad de la práctica educativa del docente y la performance estudiantil de los educandos y así lograr consolidar el logro de las competencias, de las capacidades y de las habilidades que ya poseen (Báez et al., 2017). Un programa de asistencia pedagógica virtual debe procurar analizar y explicar cuatro dimensiones importantes: la ayuda pedagógica, plataforma digital para el aprendizaje, diseño de experiencias de aprendizaje y ayuda en el análisis del procedimiento pedagógico (Altmann et al., 2021).

La teoría socio cultural de Lev Vygotsky sostiene que aún en la psicología social existe una psique individual en el ser humano que lo hace único, pero siempre proveniente de un contexto determinado. Literalmente citaba a Marx en el enunciado: "el hombre es un zoon político" entendido por Vygotsky como que somos racionalmente animales que no podemos desarrollar nuestras características ni ser "únicos" si no tenemos en nuestra existencia relaciones sociales (Vygotsky, 2006). El comportamiento de las personas no obedece a las características individuales ni a las voluntades de cada uno, sino que resulta de la interacción gregaria que mantiene con un grupo cualquiera donde se desenvuelva; de igual manera, el comportamiento del grupo no depende del comportamiento de cada uno sino nuevamente de la interacción que surja entre sus miembros (Kuz et al., 2015). Por otro lado, intentando enlazar el acompañamiento como condición humana natural con el desempeño docente debiendo ser este un facilitador para sus discentes, evocamos la 
Teoría de la No Directividad de Carl Rogers quien propugna que al ser humano no se le debe "enseñar" sino que se le debe propiciar las condiciones para su aprendizaje autónomo, o mejor aún, para su autoaprendizaje, y así garantizar verdaderos procesos cognitivos para un aprendizaje efectivo (Rogers, Carl \& Freiberg, H. Jerome, 1996). Es el maestro el que crea el lazo afectivo con sus estudiantes y su vocación de conocer más el mundo con su trato, con su actitud, con su empatía, produce en sus pupilos la misma inclinación en imitación a su preceptor, y es también la ocasión de sembrar en ellos el espíritu autónomo del investigador (Freire, 2012).

Cabe subrayar el alto relieve que Siemens (2004) ha infundido al concepto virtual, aludiendo claramente a la nueva realidad inmersa en el mundo del auge tecnológico en que todos vivimos en pleno siglo XXI, la cual nos sitúa en nuevas formas de afrontar la coyuntura actual de la pandemia producida por el COVIDSARS-2. Es importante que los docentes sepan guiar a los estudiantes en el descubrimiento de conocimientos relevantes para distinguirlos de los que no lo son; se aprende a utilizar la red para informarse y para compartir con sus compañeros los nuevos aprendizajes. La virtualidad hace posible la interacción donde a través de diálogos, preguntas y respuestas se analicen alternativas y se logren soluciones. Ello permitirá al individuo a familiarizarse con estas herramientas y con este tipo de búsqueda de soluciones (Siemens, 2004).

Y en complemento inherente a la virtualidad, la tecnología, hoy más que antes, se ha convertido en una necesidad educativa de primer nivel; se han revivido y repotenciado a las TIC que parecían obsoletas tales como la TV y la radio; y se viene desarrollando tanto en docentes como en discentes la capacidad y destreza creativa en el uso de los diversos dispositivos (García-Aretio, 2021). Ahora que existe una diversidad de aplicaciones y programas, resulta motivador para los estudiantes y maestros mejorar su desempeño en el rol que le toque fungir (Heru y Purbudi, 2021). Además, la interacción entre docentes y estudiantes permite la autorregulación de cada uno (Varela-Ordorica y ValenzuelaGonzález, 2020). Hay que destacar que las redes sociales justamente existen como producto de la interacción permanente entre las personas; de su modo de pensar, de su modo de actuar y de su modo de cambiar de actitud como resultado de esas mismas interacciones (Kuz et al., 2015).

Esta coyuntura ha obligado la adopción de nuevos trabajos en un sistema remoto inesperado lo que al mismo tiempo ha llevado a los docentes a buscar el cambio en su 
práctica pedagógica y hacer posible que el aprendizaje de las competencias no encuentren escollos (Santiago et al., 2020; Picón et al., 2020). El aislamiento social ha sorprendido y ha cambiado los hábitos en todos los sectores de educación (García-Peñalvo et al., 2020). Los distintos maestros de las instituciones educativas han sabido menguar la potencial debacle educativa reinventando nuevas estrategias y nuevos medios en cumplimiento de su misión y lo han hecho en forma extraordinaria y fundamental (Colville et al., 2021; Picón et al., 2020).

La compañía y el trabajo colaborativo entre docentes ha sido un factor primordial. La reflexión crítica supone un diálogo y una interacción entre el asistente y el acompañado para analizar la planificación y la práctica pedagógica y conseguir verdaderos datos del saber pedagógico (Agreda y Pérez, 2020). De esta manera, se subraya que la misión del asistente pedagógico es la observación y facilitación de los espacios y tiempos para dialogar sobre las actividades observadas con un propósito de mejora (Villegas et al., 2017).

La asistencia pedagógica virtual debe ser una tarea donde se construya y se comparta progresivamente los nuevos conocimientos procedimentales y que trasciendan la presencia del asistente (Morado, 2017). Es importante visualizar un diseño pedagógico que vaya acorde a las herramientas digitales y proporcionar con ello un mayor aprendizaje (Gros, 2015). Y para ello se debe contar con la asistencia de una persona que cuente con el perfil idóneo que sepa transigir ante la resistencia o temores de algunos maestros y poder transformarlas en fortalezas (Morado, 2017); influyendo significativamente en la configuración personal y gregaria de los individuos en su proceso educativo. Su calidez, empatía, motivación y compromiso puede desarrollar el espíritu crítico y retroalimentador de su par docente (García-Pérez \& Mendía, 2015).

Estamos de acuerdo en que las autoridades educativas deben propiciar un cambio y perfeccionamiento en el trabajo pedagógico de todos los docentes, de preferencia en lo que respecta a los entornos virtuales, su organización y su adecuada implementación (Fardoun et al., 2020). Y deben ir paralelo a un verdadero compromiso de vocación y dedicación que no solo motiven a los ávidos estudiantes, sino que los lleve a sentirse también comprometidos con el desarrollo de su comunidad.(Melo-Solarte \& Díaz, 2018) La asistencia pedagógica no implica necesariamente un alto desarrollo cognoscitivo y pedagógico, pero sí puede garantizar un proceso paulatino de análisis de la propia práctica 
a través del autoanálisis y de la formación de nuevos conceptos partiendo de la deconstrucción de su propia praxis (Díaz et al., 2018). La labor del docente se ve notablemente favorecida cuando su práctica pedagógica la sabe combinar con su práctica reflexiva pues así construye y transforma su propia identidad profesional (Agreda \& Pérez, 2020; Juca, 2016).

La experiencia de asistencia pedagógica virtual aparece entonces como una oportunidad de reinventar el significado del trabajo pedagógico donde la reflexión, el análisis contextual y el aprendizaje en equipo sean los pilares de esta nueva forma de conducir la formación escolar (Castro et al., 2020). Lo que no se debe dejar de lado es la reflexión permanente, mucho más en este nuevo escenario en donde la virtualidad es el principal medio de formación (Sánchez, 2020).

Por su parte, Dilma Zárate concluye en que el acompañamiento pedagógico directivo puede influir significativamente en el desempeño docente motivándolo a la observación de clases para recoger información y poder tener reuniones conjuntas y periódicas que les permita revisar las herramientas necesarias para el mejoramiento del servicio. La autora sostiene que la motivación y el trabajo del directivo fomentando el trabajo colaborativo virtual es un factor determinante para el logro de las metas (Zárate, 2021).

Gracias a todos estos estudios antecedentes, encontramos la razón por la cual la asistencia pedagógica virtual se hace necesaria para mejorar el desempeño docente sobre todo en los tiempos de pandemia. Estas investigaciones nos abren un abanico de posibilidades para continuar con la investigación de las variables que motivan este trabajo: La Asistencia Pedagógica Virtual y el Desempeño Docente.

\section{ESTRATEGIAS METODOLÓGICAS O MATERIALES Y MÉTODOS}

El tipo de estudio fue aplicado. Se desarrolló, a manera de experimento, un programa de "asistencia pedagógica virtual" para evidenciar los efectos de mejora en la variable “desempeño docente" en tiempos de pandemia. El diseño de investigación fue preexperimental y sus variables se conceptúan de la siguiente manera:

Operacionalmente, la asistencia pedagógica virtual es una estrategia social organizada para mejorar el aprendizaje de los discentes y para el fortalecimiento de la interacción y el trabajo en equipo. Permite a los involucrados hacer una reflexión del trabajo docente a partir de técnicas de observación y registro. Por otro lado, el desempeño docente es el trabajo pedagógico realizado por los maestros dentro de las aulas, previa planificación de 
sus actividades para el desarrollo de una correcta convivencia virtual con sus estudiantes y para la correcta aplicación de estrategias didácticas y de evaluación.

La muestra estuvo conformada por 31 docentes del Nivel Secundaria de la I.E. "Pedro Pablo Atusparia" de Nuevo Chimbote, Perú.

La técnica que se utilizó para recopilar la información necesaria sobre los niveles de desempeño docente fue la encuesta y se aplicó a través de un cuestionario virtual de Formulario Google, conformado por 25 ítems, antes de la aplicación del programa experimental y después del mismo. Así mismo, se recopilaron evidencias del trabajo colegiado virtual de cada docente con otros docentes y evaluaciones después de cada sesión.

El instrumento fue validado por juicio de expertos y la prueba del alfa de Cronbach le dio una confiabilidad del 0,940 después de aplicarse una prueba piloto a otros 18 docentes de otras instituciones.

Los resultados pasaron la prueba de normalidad de Shapiro y al ser comprobada su normalidad se les aplicó la prueba T de Student.

Tabla $\mathbf{N}^{\circ}$ 1. Prueba de normalidad de datos

\begin{tabular}{lccc|ccc}
\hline & \multicolumn{3}{c|}{ Kolmogorov-Smirnov $^{\text {a }}$} & \multicolumn{4}{c}{ Shapiro-Wilk } \\
\cline { 2 - 7 } & Estadístico & gl & Sig. & Estadístico & gl & Sig. \\
\hline Pre test &, 124 & 31 &, $200^{*}$ &, 933 & 31 &, 052 \\
\multirow{2}{*}{ Pos test } &, 178 & 31 &, 014 &, 842 & 31 &, 000 \\
\hline
\end{tabular}

*. Esto es un límite inferior de la significación verdadera.

a. Corrección de significación de Lilliefors

Fuente: Base de datos estadísticos

De los datos obtenidos en el estudio se tiene la prueba de normalidad de datos donde se sustenta la distribución de la muestra, siendo analizada por medio del método de Shapiro Wilk para muestras inferiores a 50, obteniendo un valor de significancia de 0.052 para el pre test y de 0.000 para el pos test; al realizar el análisis se puede evidenciar que el valor más alto se ubica en 0.052 , considerado como superior al 0.05 , por lo cual se toma la decisión de que la muestra presenta una distribución paramétrica, por lo tanto el método para hallar la hipótesis es el método de T de Student. 
Tabla 2. Prueba T de Student para comprobar la hipótesis

\begin{tabular}{lccccc}
\hline \multirow{2}{*}{ Variable } & $\begin{array}{c}\text { Valor } \\
\text { observado }\end{array}$ & $\begin{array}{c}\text { Prueba T - Student } \\
\text { Valor } \\
\text { tabular }\end{array}$ & $\begin{array}{c}\text { Probabilidad } \\
\text { significancia }\end{array}$ & $\begin{array}{c}\text { Nivel de } \\
\text { significancia }\end{array}$ & $\begin{array}{c}\text { Decisión } \\
\mathbf{t}_{\mathbf{0}}>\mathbf{t}_{\mathbf{c}} \\
\mathbf{p}<\alpha\end{array}$ \\
\hline $\begin{array}{l}\text { Desempeño } \\
\text { docente }\end{array}$ & $\mathrm{t}_{\mathrm{o}}=8,612$ & $\mathrm{t}_{\mathrm{c}}=1,697$ & $\mathrm{p}=0,0000$ & $\alpha=$ & Se rechaza \\
& & & 0,05 & $H_{0}$ \\
\hline
\end{tabular}

Fuente: Base de datos estadísticos.

Al realizar el análisis de la prueba de hipótesis se tiene la prueba T de Student; para ello, se ha logrado obtener un valor T observado de 8,612 considerado como superior al valor T tabular 1,697 (30 grados de libertad), con lo cual se considera que existe incidencia de la asistencia pedagógica virtual sobre el desempeño docente, además al analizar el valor de la significancia obtenida se tiene un valor de 0.000 , situado por debajo del 0.05 con lo cual se procede al rechazo de la hipótesis nula y se comprueba la hipótesis de estudio afirmando que la asistencia pedagógica virtual mejora significativamente el desempeño docente en tiempos de pandemia en la I.E. Pedro Pablo Atusparia de Nuevo Chimbote.

\section{RESULTADOS Y DISCUSIÓN}

Si el objetivo general fue demostrar que la asistencia pedagógica virtual mejora significativamente el desempeño docente en tiempos de pandemia en la I.E. PPA de Nuevo Chimbote en 2021, se tiene que para el nivel deficiente en el pre test no se registraron estudiantes, luego en el post test tampoco se registraron estudiantes; luego, al realizar el análisis del nivel regular, se obtuvo en el pre test a 10 estudiantes (32.3\%), que en comparación del post test no se registraron estudiantes, logrando obtener una diferencia del $32.3 \%$, luego en el nivel eficiente se obtuvo en el pre test a 21 estudiantes (67.7\%), que en comparación del post test en donde se obtuvo a 31 estudiantes (100.0\%), se logró una diferencia del $32.3 \%$, como podemos observar en la siguiente tabla:

Tabla 3. Mejoramiento del desempeño docente en tiempos de pandemia gracias a la asistencia pedagógica virtual.

\begin{tabular}{ccccccc}
\hline Niveles & \multicolumn{2}{c}{ Pre Test } & \multicolumn{2}{c}{ Post Test } & \multicolumn{2}{c}{ Diferencia } \\
& $\mathbf{f i}$ & $\mathbf{\%}$ & $\mathbf{f i}$ & $\mathbf{\%}$ & $\mathbf{f i}$ & $\mathbf{\%}$ \\
\hline Deficiente & 0 & $0.0 \%$ & 0 & $0.0 \%$ & 0 & $0.0 \%$ \\
Regular & 10 & $32.3 \%$ & 0 & $0.0 \%$ & 10 & $32.3 \%$ \\
Eficiente & 21 & $67.7 \%$ & 31 & $100.0 \%$ & 10 & $32.3 \%$ \\
Total & 31 & $100.0 \%$ & 31 & $100.0 \%$ & & \\
\hline
\end{tabular}

Fuente: Base de datos de pre test y post test 
Con ello se pudo demostrar la efectividad de la asistencia pedagógica virtual pues mejoró significativamente el desempeño docente en tiempos de pandemia en la I.E. Pedro Pablo Atusparia de Nuevo Chimbote, confirmándose así la hipótesis de investigación.

Este resultado permite confirmar la teoría del aprendizaje social de Bandura, la misma que señala que una persona puede aprender siguiendo el modelado de otra y a la inspiración que le produce (Bandura, 1975). También se confirma la Teoría Sociocultural de Vygostsky quien sostiene que la zona de desarrollo potencial puede ser alcanzado por un aprendiz siempre que tenga el apoyo y compañía de otra persona que sepa guiarlo. Estos resultados también concuerdan con la investigación de Varela (2019) quien afirma que la asistencia pedagógica con herramientas virtuales optimizará el desempeño docente y dará a los maestros mayor seguridad. Y se confirma también que el asistente pedagógico influye significativamente en la formación personal y social del docente en el proceso educativo (García-Pérez y Mendia, 2015). Sin embargo, oficialmente, en las distintas instituciones educativas no se encuentra generalizado este programa de asistencia dando advertencia, de esta manera, que aún muchos docentes que no conocen el mundo virtual carecen de las herramientas mínimas para mejorar su desempeño en tiempos de pandemia. Nuestras autoridades y funcionarios del sector educativo (y demás sectores) deben sacar provecho del avance y perfeccionamiento de las TIC para lo cual deben gestionar su más pronta implementación para reducir las limitaciones en los estudiantes y maestros (Heru \& Purbudi, 2021). Ya parecían proféticas esas palabras que avizoraban en estos tiempos, que ya se vislumbran escenarios distintos a los tradicionales y con impactantes cambios para el escenario educativo, sobre todo, con la seguridad de que las nuevas TIC llegan para posicionarse en forma muy sólida especialmente en este ámbito (Kuz et al., 2015). El primer objetivo específico era evidenciar que el Programa de Asistencia Pedagógica Virtual mejora las tareas de Planificación de los docentes.

Tabla 4. Resultados del programa APEVI para mejorar las tareas de Planificación Docente.

\begin{tabular}{ccccccc}
\hline Niveles & \multicolumn{2}{c}{ Pre Test } & \multicolumn{2}{c}{ Post Test } & \multicolumn{2}{c}{ Diferencia } \\
& $\mathbf{f i}$ & $\mathbf{\%}$ & $\mathbf{f i}$ & $\mathbf{\%}$ & $\mathbf{f i}$ & $\mathbf{\%}$ \\
\hline Deficiente & 0 & $0.0 \%$ & 0 & $0.0 \%$ & 0 & $0.0 \%$ \\
Regular & 10 & $32.3 \%$ & 1 & $3.2 \%$ & 9 & $29.0 \%$ \\
Eficiente & 21 & $67.7 \%$ & 30 & $96.8 \%$ & 9 & $29.0 \%$ \\
Total & 31 & $100.0 \%$ & 31 & $100.0 \%$ & & \\
\hline
\end{tabular}

Fuente: Base de datos de datos estadísticos 
De los resultados presentados en el estudio se tiene que para el Nivel Deficiente en el pre test no se registró estudiantes, luego en el post test tampoco hubo estudiantes; luego al realizar el análisis del Nivel Regular se obtuvo en el pretest a 10 estudiantes (32.3\%), que en comparación del post test se registró a 1 estudiante (3.2\%), logrando obtener una diferencia del 29.0\%; luego en el Nivel Eficiente se obtuvo en el pretest a 21 estudiantes (67.7\%), que en comparación del post test se obtuvo a 31 estudiantes (100.0\%), haciendo una diferencia del 32.3\%. Con ello se pudo demostrar la efectividad que presentó la asistencia pedagógica virtual pues mejora significativamente las tareas de planificación docente en tiempos de pandemia en la I.E. Pedro Pablo Atusparia de Nuevo Chimbote. Al respecto, Báez et al. (2016) sostienen que la asistencia pedagógica centra la posición del docente como ente motivador capaz de organizar actividades más eficaces tanto metodológicas como evaluativas y con la investigación hecha se confirma este aporte. De igual manera, Valdivia (2016) en su tesis recalca que la asistencia pedagógica contribuye de forma positiva con el trabajo docente en las diversas dimensiones que abarca esta variable (I. S. Valdivia, 2016). Se logró corroborar que los planes de estudios y sus respectivas implicancias deben ser revisados y actualizados en las distintas unidades de gestión educativa locales, de acuerdo a las necesidades y a la realidad que viven los educandos y sus respectivas familias, así como el contexto donde está inmersa la institución educativa (Garbe et al., 2020). Sin embargo, no existe una norma que oficialice y garantice la realización de una asistencia pedagógica virtual y queda en manos de los directivos el afianzamiento de esta estrategia pues hay temas fundamentales al analizar el desempeño de un profesor como la planificación curricular basada en las características contextuales de los discentes, el desarrollo de las sesiones con el uso pertinente de estrategias didácticas y la evaluación, especialmente formativa en tiempos de pandemia (Cotaquispe et al., 2021).

El segundo objetivo específico era evidenciar que el Programa de Asistencia Pedagógica Virtual mejora la convivencia virtual con los estudiantes en la I.E. Pedro Pablo Atusparia, 2021. 
Tabla 5. Resultados del programa APEVI en mejora de la convivencia virtual con los estudiantes.

\begin{tabular}{ccccccc}
\hline \multirow{2}{*}{ Niveles } & \multicolumn{2}{c}{ Pre Test } & \multicolumn{2}{c}{ Post Test } & \multicolumn{2}{c}{ Diferencia } \\
& $\mathbf{f i}$ & $\mathbf{\%}$ & $\mathbf{f i}$ & $\mathbf{\%}$ & $\mathbf{f i}$ & $\mathbf{\%}$ \\
\hline Deficiente & 0 & $0.0 \%$ & 0 & $0.0 \%$ & 0 & $0.0 \%$ \\
Regular & 4 & $12.9 \%$ & 0 & $0.0 \%$ & 4 & $12.9 \%$ \\
Eficiente & 27 & $87.1 \%$ & 31 & $100.0 \%$ & 4 & $12.9 \%$ \\
Total & 31 & $100.0 \%$ & 31 & $100.0 \%$ & & \\
\hline
\end{tabular}

Fuente: Base de datos de datos estadísticos

De los resultados presentados en el estudio se tiene que para el Nivel Deficiente en el pre test no se registró estudiantes, luego en el post test tampoco hubo estudiantes; luego, al realizar el análisis del Nivel Regular se obtuvo en el pretest a 4 estudiantes (12.9\%), que en comparación del post test se registró 0 estudiantes $(0.0 \%)$, logrando obtener una diferencia del $12.9 \%$, luego en el Nivel Eficiente se obtuvo en el pretest a 27 estudiantes (87.1\%), que en comparación del post test se obtuvo a 31 estudiantes (100.0\%), haciendo una diferencia del $12.9 \%$. Con ello se pudo demostrar la efectividad que presentó la asistencia pedagógica virtual pues mejora significativamente la convivencia virtual en tiempos de pandemia en la I.E. Pedro Pablo Atusparia de Nuevo Chimbote. Al respecto, Freire (2012) afirmaba que es el maestro el que crea el lazo afectivo con sus estudiantes y su vocación de conocer más el mundo con su trato, con su actitud, con su empatía, produce en sus pupilos la misma inclinación en imitación a su preceptor. Se confirma que la asistencia pedagógica implica una relación de calidad donde el maestro sepa transmitir calidez, empatía, motivación y compromiso por el vigor del proceso educativo en un ambiente donde la verdad y el respeto fortalezca la autoestima y la autonomía de los verdaderos protagonistas (García-Pérez \& Mendía, 2015). Incluso, la presencia importante de los distintos tutoriales encontrados en internet no iguala a la importancia de la enseñanza virtual ejercida por los maestros. La razón principal es la interacción misma, si es con cámara encendida es mucho mejor, y no tiene límites de tiempo o espacio (Tseng \& Chen, 2020). Últimos estudios han descubierto lo significativo que resulta la configuración de comunidades virtuales de aprendizaje profesional para compartir, informar y evaluar tal como hacían los maestros antes de la cuarentena. No hay que olvidar que este ejercicio no solo debe involucrar a discentes sino también a sus progenitores pues toca a los docentes ser parte de sus limitaciones y aspiraciones (Garbe et al., 2020). El docente en su desempeño virtual tiene la responsabilidad y el compromiso 
de diseñar espacios y momentos virtuales de aprendizaje que aseguren la sana convivencia y sobre todo la salud mental de sus discentes teniendo en cuenta que el contexto pandémico actual ha consternado y desmotivado a muchos de ellos (Shah et al., 2021). Sobre todo, en estos tiempos de pandemia, el profesor debe practicar con cariño la pedagogía de la palabra, el coloquio y la escucha activa, no solo con sus pupilos sino también con los respectivos progenitores para que la guía y el soporte socioemocional que le corresponde se dé de forma efectiva y fructífera (Herrera-García et al., 2021).

El tercer objetivo específico era evidenciar que el Programa de Asistencia Pedagógica Virtual mejora la estrategia didáctica de los docentes.

Tabla 6. Resultados del programa APEVI en mejora de la Estrategia Didáctica del docente.

\begin{tabular}{ccccccc}
\hline Niveles & \multicolumn{2}{c}{ Pre Test } & \multicolumn{2}{c}{ Post Test } & \multicolumn{2}{c}{ Diferencia } \\
& $\mathbf{f i}$ & $\mathbf{\%}$ & $\mathbf{f i}$ & $\mathbf{\%}$ & $\mathbf{f i}$ & $\mathbf{\%}$ \\
\hline Deficiente & 0 & $0.0 \%$ & 0 & $0.0 \%$ & 0 & $0.0 \%$ \\
Regular & 10 & $32.3 \%$ & 2 & $6.5 \%$ & 8 & $25.8 \%$ \\
Eficiente & 21 & $67.7 \%$ & 29 & $93.5 \%$ & 8 & $25.8 \%$ \\
Total & 31 & $100.0 \%$ & 31 & $100.0 \%$ & & \\
\hline
\end{tabular}

Fuente: Base de datos estadísticos

De los resultados presentados en el estudio se tiene que para el nivel deficiente en el pre test no se registró estudiantes, luego en el post test tampoco se registraron estudiantes, luego al realizar el análisis del nivel regular se obtuvo en el pre test a 10 estudiantes (32.3\%), que en comparación del post test se registró 2 estudiantes (6.5\%), logrando obtener una diferencia del $25.8 \%$, luego en el nivel eficiente se obtuvo en el pre test a 21 estudiantes (67.7\%), que en comparación del post test se obtuvo a 29 estudiantes (93.5\%), haciendo una diferencia del $25.8 \%$. Con ello se pudo demostrar la efectividad que presentó la asistencia pedagógica virtual pues mejora significativamente la estrategia didáctica en tiempos de pandemia en la I.E. Pedro Pablo Atusparia de Nuevo Chimbote. Al respecto, Ágreda y Pérez (2020) sostienen que la asistencia pedagógica permite una actualización docente en base al diálogo colegiado con otros docentes sobre su quehacer didáctico y con nuevos saberes necesarios en el grupo profesional de maestros. Nuestro ejercicio pedagógico vuelve a empezar, pero ahora con el compromiso de buscar el reencuentro entre todos los agentes educativos; la asistencia pedagógica se erige como la principal estrategia para superar juntos este aislamiento obligatorio (Gerbaldo y Granato, 
2020). Se ha podido constatar a través de varias evidencias de que los docentes no solo están transformando su estrategia didáctica, sino que hasta se están reinventando ellos mismos tratando de cumplir su noble tarea de formación de niños y adolescentes (Colville et al., 2021). Hay que tomar en consideración que mientras la sociedad en general, donde están inmersos tanto estudiantes como maestros, se está preparando y/o adaptando a una nueva forma de aprender y de interactuar, mediante las sesiones de clase virtuales, es menester incluir en las programaciones educativas las mayores estrategias de aprendizaje online en las nuevas formas educativas que se avecinan (Hsiao, 2021). Después de los resultados obtenidos en numerosos estudios similares, se ha podido notar que la labor de un profesor radica básicamente en planificar, motivar, dominar las estrategias pedagógicas y el uso acertado de herramientas principalmente tecnológicas (Cotaquispe et al., 2021). Además, hay que destacar que siempre la dimensión referida a la estrategia didáctica se constituye como uno de los pilares vertebrales para el logro de las competencias, además de las competencias transversales de los discentes, vale decir, aquellas centradas en su autonomía para aprender, y en su interrelación con los escenarios de la virtualidad (Digión y Álvarez, 2021).

El cuarto objetivo específico era evidenciar que el Programa de Asistencia Pedagógica Virtual mejora la estrategia de evaluación de los docentes.

Tabla 7. Resultados del programa APEVI en mejora de la estrategia de evaluación.

\begin{tabular}{ccccccc}
\hline Niveles & \multicolumn{2}{c}{ Pre Test } & \multicolumn{2}{c}{ Post Test } & \multicolumn{2}{c}{ Diferencia } \\
& $\mathbf{f i}$ & $\mathbf{\%}$ & $\mathbf{f i}$ & $\mathbf{\%}$ & $\mathbf{f i}$ & $\mathbf{\%}$ \\
\hline Deficiente & 1 & $3.2 \%$ & 0 & $0.0 \%$ & 1 & $3.2 \%$ \\
Regular & 11 & $35.5 \%$ & 3 & $9.7 \%$ & 8 & $25.8 \%$ \\
Eficiente & 19 & $61.3 \%$ & 28 & $90.3 \%$ & 9 & $29.0 \%$ \\
Total & 31 & $100.0 \%$ & 31 & $100.0 \%$ & & \\
\hline
\end{tabular}

Fuente: Base de datos estadísticos

De los resultados presentados en el estudio se tiene que para el nivel deficiente se obtuvo en el pre test a 1 estudiante (3.2\%), que en comparación del post test se registró 0 estudiantes $(0.0 \%)$, logrando obtener una diferencia del 3.2\%, luego al realizar el análisis del nivel regular se obtuvo en el pre test a 11 estudiantes (35.5\%), que en comparación del post test se registró 3 estudiantes (9.7\%), logrando obtener una diferencia del $25.8 \%$, luego en el nivel eficiente se obtuvo en el pre test a 19 estudiantes (61.3\%), que en comparación del post test se obtuvo a 28 estudiantes (90.3\%), haciendo una diferencia del $29.0 \%$. Con ello se pudo demostrar la efectividad que presentó la asistencia 
pedagógica virtual pues mejora significativamente la estrategia de evaluación en tiempos de pandemia en la I.E. Pedro Pablo Atusparia de Nuevo Chimbote. Al respecto, si el docente va a evaluar online es importante que conozca y domine las herramientas virtuales para ejercer una tarea evaluadora y retroalimentadora en línea así como ser capaz de innovar en las estrategias de evaluación (Fardoun et al., 2020). No se debe olvidar que la asistencia pedagógica sustenta la reflexión docente y la reflexión docente que ejerce un profesor antes, durante y después de su actividad pedagógica lo lleva a observar con mayor objetividad su praxis y por ende, lo lleva a ser más analítico y autocrítico como facilitador del proceso pedagógico en sus discentes. En esta nueva era de la información y de la virtualidad educativa, la globalización se constituye como uno de los procesos principales que la caracteriza y, más aún, son los estudiantes, niños y jóvenes, los más sensibles ante los avances vertiginosos de la tecnología innovadora y, como tal, propensos a los cambios nuevos de la personalidad "cibersocial". Frente a esta amenaza cibernética, el docente mejor preparado en herramientas digitales estará en mejores condiciones de evaluar, entender y guiar el camino de sus discentes (Ryzhanova et al., 2021). Hay temas fundamentales al analizar el desempeño de un profesor como la planificación curricular basada en las características contextuales de los discentes, el desarrollo de las sesiones con el uso pertinente de estrategias didácticas y la evaluación, especialmente formativa en tiempos de pandemia (Cotaquispe et al., 2021). Gracias a la asistencia pedagógica, la reflexión del maestro sobre su propia práctica puede tener como efecto la capacidad autónoma y resolutiva de transformar su ejercicio docente y elevar la calidad de su desempeño y así generar también estudiantes reflexivos de su propio aprendizaje (Agreda \& Pérez, 2020). Por eso, el Director de la institución educativa debe saber motivar y estimular la asistencia pedagógica virtual a sus docentes, y tampoco hay que olvidar que es importante devolver al maestro observado las indicaciones necesarias para mejorar sus posibles falencias en el trabajo pedagógico con el fin de elevar la calidad de su praxis de acuerdo a las necesidades de sus pupilos y a las características del contexto (Vega \& Vasquez, 2021).

\section{CONCLUSIONES}

- De acuerdo al objetivo general, se ha llegado a la conclusión de que la asistencia pedagógica virtual mejora significativamente el desempeño docente en tiempos de pandemia en la I.E. Pedro Pablo Atusparia de Nuevo Chimbote, es decir, que este 
acompañamiento se convierte en una estrategia fundamental para motivar, orientar y complementar la experiencia y conocimiento del maestro, a fin de que este pueda responder con aptitudes y acciones a las expectativas de educandos y padres en la nueva educación virtual que ya ganó posicionamiento.

- De acuerdo al primer objetivo específico, se pudo validar el programa de asistencia pedagógica virtual para mejorar el desempeño docente en tiempos de pandemia en la I.E. PPA de Nuevo Chimbote.

- De acuerdo al tercer objetivo específico, se pudo determinar el nivel de desempeño docente en tiempos de pandemia en la I.E. PPA de Nuevo Chimbote antes y después de la aplicación del programa.

- De acuerdo al tercer objetivo específico, se logró demostrar que la asistencia pedagógica virtual mejoró significativamente las tareas de planificación docente en tiempos de pandemia en la I.E. PPA de Nuevo Chimbote.

- De acuerdo al cuarto objetivo específico, se pudo demostrar que la asistencia pedagógica virtual mejoró significativamente la convivencia virtual entre docentes, y entre docentes y estudiantes en tiempos de pandemia en la I.E. PPA de Nuevo Chimbote.

- De acuerdo al quinto objetivo específico, se pudo demostrar que la asistencia pedagógica virtual mejoró significativamente la estrategia didáctica del docente en tiempos de pandemia en la I.E. PPA de Nuevo Chimbote.

- De acuerdo al sexto objetivo específico, se pudo demostrar que la asistencia pedagógica virtual mejoró significativamente la estrategia de evaluación del docente en tiempos de pandemia en la I.E. PPA de Nuevo Chimbote.

\section{LISTA DE REFERENCIAS}

Agreda, A., \& Pérez, M. (2020). Relación entre acompañamiento pedagógico y práctica reflexiva. Revista de Educación, 2(30), 219-232. http://sedici.unlp.edu.ar/handle/10915/54608

Aguayo-Rousell, H. B. (2020). Personal formador de docentes. Representaciones sociales de su profesión. Revista Electrónica Educare, 24(1), 1-22. https://doi.org/10.15359/ree.24-1.18

Aguilar, D. (2015). Ser docente virtual: tiempo y presencia en entornos de enseñanzaaprendizaje del campus Andaluz Virtual. Un estudio de caso [Universidad de 
Málaga]. https://dialnet.unirioja.es/servlet/tesis?codigo=78971

Altmann, M., Clauss, A., \& Schoop, E. (2021). Virtual collaborative learning during COVID19 - Lessons learned in practice. IDIMT 2021 - Pandemics: Impacts, Strategies and Responses, 29th Interdisciplinary Information Management Talks, 467-474.

Aramayo, A., López, A., Díaz, R., \& Astorga, F. (2020). Acompañar las prácticas educativas virtuales en tiempos de pandemia. In Pensando la pandemia en /desde Jujuy reflexiones situadas (pp. 15-21).

AUCCA, J. (2021). Monitoreo, acompañamiento pedagógico y clima institucional en el contexto del COVID-19 de la Gran Unidad Escolar Inca Garcilaso de la Vega del distrito de Cusco - 2020. In UNIVERSIDAD NACIONAL DE EDUCACIÓN Enrique Guzmán y Valle. Universidad Nacional de Educación.

Báez, J. R., Benavidez Briones, W. Y., Gómez Calero, G. Y., González Olivas, N. A., \& Castillo Herrera, B. (2017). Caracterización del programa de acompañamiento pedagógico en el marco del Plan Nacional de Desarrollo Humano (PNUD) de Nicaragua. Revista Científica de FAREM-Estelí, 5-17. https://doi.org/10.5377/farem.v0i0.3108

Bandura, A. (1975). Análisis del aprendizaje social de la agresión. In Trillas (Ed.), Blog de Psicoactiva (pp. 308-351).

Bansak, C., \& Starr, M. (2020). Covid-19 shocks to education supply: how 200,000 U.S. households dealt with the sudden shift to distance learning. Review of Economics of the Household, 19, 63-90. https://doi.org/10.1007/s11150-020-09540-9

Carrasco, M. E. (2020). Efectos del programa Acompeda en el desempeño pedagógico de los docentes en instituciones educativas de Tumbes, 2019. César Vallejo.

Castro, H., Arguedas Quesada, C., \& Cortés, K. R. (2020). Acompañamiento pedagógico del Programa de Tecnologías para el Aprendizaje [Protea]: Una experiencia constructivista que aprovecha el Makey Makey y Scratch para enriquecer un curso de Expresión Musical. Revista Educación, 44. https://doi.org/10.15517/revedu.v44i2.39179

Colville, T., Hulme, S., Kerr, C., Mercieca, D., \& Mercieca, D. P. (2021). Teaching and Learning in COVID-19 Lockdown in Scotland: Teachers' Engaged Pedagogy. Frontiers in Psychology, 12. https://doi.org/10.3389/FPSYG.2021.733633 
Cornejo, W. (2020). Modelo de gestión de acompañamiento pedagógico directivo para el desarrollo del pensamiento crítico del docente de la Institución Educativa 11017 [Universidad César Vallejo]. In Repositorio Institucional - UCV. http://repositorio.ucv.edu.pe/handle/20.500.12692/49635\#.XFmX8_M5nY.mendeley

Cotaquispe, L., Soplapuco, J., Rivas, A., \& Vales, J. (2021). Revisión sistemática del desempeño docente en la educación. Revista Iberoamericana de La Educación, 1 .

Díaz, M., García, J., \& Legañoa, M. (2018). Modelo de gestión del acompañamiento pedagógico para maestro de primaria. Transformación, 14(1), 44-57.

Digión, L. B., \& Álvarez, M. M. (2021). Teaching and learning experience with a virtual classroom in the field of pedagogical support due to Covid-19. Apertura, 13(1), 20-35. https://doi.org/10.32870/ap.v13n1.1957

Espinosa-Izquierdo, J. G., Morán-Peña, F. L., \& Granados-Romero, J. F. (2021). El Síndrome Burnout y su efecto en el desempeño docente en tiempo de pandemia. Polo Del Conocimiento, 6, 670-679. https://doi.org/10.23857/pc.v6i3.2395

Fardoun, H., González, C., Collazos, C., \& Yousef, M. (2020). Estudio exploratorio en Iberoamérica sobre procesos de enseñanza-aprendizaje y propuesta de evaluación en tiempos de pandemia Habib. Education in the Knowledge Society, 21, 1-9.

Freire, P. (2012). Pedagogía de la autonomía: Saberes necesarios para la práctica educativa. https://es.scribd.com/read/370352773/Pedagogia-de-la-autonomiaSaberes-necesarios-para-la-practica-educativa

Galaz, A., Jiménez Vásquez, M. S., \& Díaz-Barriga, Á. (2019). Evaluación del desempeño docente en Chile y México. Perfiles Educativos, XLI(163), 177-199. http://www.scielo.org.mx/pdf/peredu/v41n163/0185-2698-peredu-41-163177.pdf

Garbe, A., Ogurlu, U., Logan, N., \& Cook, P. (2020). COVID-19 and Remote Learning: Experiences of Parents with Children during the Pandemic. American Journal of Qualitative Research, 4(3), 45-65. https://doi.org/10.29333/ajqr/8471

García-Aretio, L. (2021). Radio, televisión, audio y vídeo en educación. Funciones y posibilidades, potenciadas por el COVID-19. RIED. Revista Iberoamericana de Educación a Distancia, 25(1), 31468. https://doi.org/10.5944/ried.25.1.31468

García-Peñalvo, F. J., Corell, A., Abella-García, V., \& Grande, M. (2020). La evaluación 
online en la educación superior en tiempos de la COVID-19. Education in the Knowledge Society, 21, 1-26. https://doi.org/10.14201/eks.23013

García-Pérez, Á., \& Mendía, R. (2015). Acompañamiento Educativo: El rol del educador en Aprendizaje y Servicio Solidario. Profesorado: Revista de Currículum y Formación Del Profesorado, 19, 42-58.

Gerbaldo, G., \& Granato, P. (2020). El acompañamiento en tiempos de pandemia: ¿una invitación la reflexión? (pp. 161-165). https://ri.conicet.gov.ar/handle/11336/122556

Gomero, N. (2020). Plan de monitoreo integral pedagógico en el desempeño del docente de Educación Básica Regular, Ancash, 2019 (Vol. 2507, Issue February).

Gros, B. (2015). La caída de los muros del conocimiento en la sociedad digital y las pedagogías emergentes. Education in the Knowledge Society (EKS), 16, 58-68. https://doi.org/10.14201/eks20151615868

Hernández Sampieri, R., Fernández Collado, C., \& Baptista Lucio, P. (2014). Metodología de la investigación $\left(6^{\circ}\right)$.

Herrera-García, R. E., Vargas-Castillo, A. M., Martínez-Ibarra, M. M., \& RodríguezMontes, Á. B. (2021). Desempeño digital de los docentes desde la emergencia sanitaria del COVID-19. Revista Arbitrada Interdisciplinaria KOINONIA, VI, $763-779$.

Heru, K., \& Purbudi, W. (2021). Do Electronic Information Resources Improve Student Academic Performance? Case at the Student of Management Study Program in Yogyakarta Indonesia. Technium Social Sciences Journal, 24, 460-467.

Hsiao, Y. C. (2021). Impacts of course type and student gender on distance learning performance: A case study in Taiwan. Education and Information Technologies, 26(6), 6807-6822. https://doi.org/10.1007/s10639-021-10538-8

Huaman, L. (2021). Educación remota y desempeño docente en las instituciones educativas de educación secundaria en el distrito de Huancavelica en tiempos de Covid-19. In Repositorio Institucional - UCV.

Huamani, T. E., Valencia Lima, L. R., \& Ramírez Rosales, F. G. (2019). Programa EDUSOFT y desempeño docente en las instituciones educativas públicas del nivel secundario. Revista de Investigación Puriq, 1, 240-252.

Juca, F. (2016). La educación a distancia, una necesidad para la formación de los 
profesionales. Revista Universidad y Sociedad, 8(1), 106-111. http://scielo.sld.cu/pdf/rus/v8n1/rus15116.pdf

Kuz, A., Falco, M., Nahuel, L., \& Giandini, R. (2015). Agent SocialMetric: herramienta de asistencia al docente para determinar el clima social y la estructura del aula. $I E$ Comunicaciones: Revista Iberoamericana de Informática Educativa, 22, 16-29. https://dialnet.unirioja.es/servlet/articulo?codigo=5162179

Lau, E. (2020). Modelo de gestión virtual para mejorar el proceso de acompañamiento en la unidad de gestión educativa local de Lambayeque. In Universidad César Vallejo.

Lozada, C. (2018). Modelo de gestión pedagógica de monitoreo y acompañamiento para fortalecer el desempeño docente en Instituciones Públicas del Nivel Primario del Distrito de Puerto Eten - 2016. In Universidad César Vallejo.

Martínez Chairez, G. I., Guevara Araiza, A., \& Valles Ornelas, M. M. (2016). El desempeño docente y la calidad educativa. $R a$ Ximhai, 123-134. https://doi.org/10.35197/rx.12.01.e3.2016.06.gm

Melo-Solarte, D. S., \& Díaz, P. A. (2018). El Aprendizaje Afectivo y la Gamificación en Escenarios de Educación Virtual. Informacion Tecnologica, 29(3), 237-248. https://doi.org/10.4067/S0718-07642018000300237

Meza, L. F., Torres Miranda, J. S., \& Mamani-Benito, O. (2021). Gestión educativa como factor determinante del desempeño de docentes de educación básica regular durante la pandemia Covid-19, Puno-Perú. Apuntes Universitarios, 11(1), 23-35. https://doi.org/10.17162/au.v11i1.543

Morado, M. F. (2017). El acompañamiento tecno-pedagógico como alternativa para la apropiación de tecnología en docentes universitarios. Actualidades Investigativas En Educación, 17(3). https://doi.org/10.15517/aie.v17i3.29688

Picón, G. A., González de Caballero, G. K., \& Paredes Sánchez, J. N. (2020). Desempeño y formación docente en competencias digitales en clases no presenciales durante la pandemia COVID-19. Scielo Preprints, 1-16. https://preprints.scielo.org/index.php/scielo/preprint/view/778

Revilla-Mendoza, J. T., \& Palacios-Jimenez, A. S. (2020). Ser Docente En Tiempos De Pandemia Por Covid-19: Evaluación Del Desempeño Docente En Una Universidad Pública De Lima. Revista de Investigación Científica Ágora, 7(2), 
Barreno Flores...

58-62. https://doi.org/10.21679/arc.v7i2.197

Rojas, R. A., \& Cachay, H. G. (2021). Desempeño Docente Ante La Enseñanza Virtual En Escenarios Pandémicos. Revista Cientifica Epistemia, 5(1). https://doi.org/10.26495/re.v5i1.1886

Ryzhanova, A. O., Potomkina, N. Z., \& Polyanichko, A. O. (2021). High School Students' Dependence on Virtual Social Networks: Approaches to SocioPedagogical Prevention in Ukraine. Journal of Educational and Social Research, 11(4). https://doi.org/10.36941/jesr-2021-0085

Salvador, R. (2021). Polya y el acompañamiento pedagógico en Ventanilla-Callao 2020. http://repositorio.uncp.edu.pe/bitstream/handle/UNCP/3000/Silva Acosta.pdf?sequence=1\&isAllowed=y\%0Ahttps://repositorio.comillas.edu/xmlu i/handle/11531/1046

Sánchez, P. J. (2020). Influencia del liderazgo directivo en el desempeño docente, Virú 2020. César Vallejo.

Santiago, B., Scorsolini, F., \& Barcellos, R. de C. de M. (2020). Ser docente en el contexto de la pandemia de COVID-19: reflexiones sobre la salud mental. Index de Enfermería, 29 , $137-141$. https://scielo.isciii.es/scielo.php?script=sci_arttext\&pid=S1132-

$12962020000200008 \& \operatorname{lng}=e s \& n r m=i s o \& t \operatorname{lng}=e s$

Schultz, D., \& Schultz, S. (2010). Teorías de la Personalidad.

Shah, S. S., Shah, A. A., Memon, F., Kemal, A. A., \& Soomro, A. (2021). Online learning during the COVID-19 pandemic: Applying the self-determination theory in the 'new normal.' Revista de Psicodidáctica, 26(2), 169-178. https://doi.org/10.1016/j.psicod.2020.12.004

Sianes, A., \& Sánchez, E. (2020). E-learning en 15 días. Retos y renovaciones en la Educación Primaria y Secundaria de la República de Croacia durante la crisis del COVID-19. How have we Introduced distance Learning? Revista Española de Educación Comparada, 36, 181-195. https://doi.org/10.5944/reec.36.2020.27637

Siemens, G. (2004). Conectivismo: una teoria de aprendizaje para la era digital. $\begin{array}{llllll}\text { Conectados En } & \text { El } & \text { Ciberespacio, } & 5, & 10 .\end{array}$ https://s3.amazonaws.com/academia.edu.documents/38778149/13_conectivismo _era_digital.pdf?AWSAccessKeyId=AKIAIWOWYYGZ2Y53UL3A\&Expires= 
1549576205\&Signature=E0xTaLrGSAXyOZi0cCe\%2Bk\%2FgqlSQ\%3D\&resp onse-content-disposition=inline \%3B filename\%3DEste_trabajo_est

Tamay-Chimborazo, L. U., García-Herrera, D. G., Cabrera-Berrezueta, L. B., \& ErazoÁlvarez, J. C. (2020). Aprovechamiento de las Tecnologías Educativas por los docentes en tiempos de pandemia. Cienciamatria, 6(3), 4-28. https://doi.org/10.35381/cm.v6i3.388

Tinoco, C. A. (2020). Liderazgo y acompañamiento pedagógico del Equipo Directivo en las Instituciones Educativas de Jornada Escolar Completa. Ancash, 2019 [Universidad César Vallejo]. http://dx.doi.org/10.1016/j.ndteint.2014.07.001\%0Ahttps://doi.org/10.1016/j.ndt eint.2017.12.003\%0Ahttp://dx.doi.org/10.1016/j.matdes.2017.02.024

Tseng, C. J., \& Chen, T. C. (2020). Impact of web-based teaching on the learning performance of education and training in the service industry during COVID-19. Contemporary Educational Technology, $12(2), \quad 1-8$. https://doi.org/10.30935/cedtech/8581

Valdivia, C. (2015). Modelo de monitoreo y asesoramiento pedagógico para mejorar el desempeño docente en educación secundaria - Chiclayo - 2015. In Universidad César Vallejo. César Vallejo.

Valdivia, I. S. (2016). Influencia del acompañamiento pedagógico en el desempeño docente en la Institución Educativa Emblemática "Divina Pastora", Oxapampa, Pasco-2016. In Universidad César Vallejo. César Vallejo.

Varela-Ordorica, S. A., \& Valenzuela-González, J. R. (2020). Uso de las tecnologías de la información y la comunicación como competencia transversal en la formación inicial de docentes. Revista Electrónica Educare, 24, 1-20. https://doi.org/10.15359/ree.24-1.10

Vásquez, C. E., \& Henríquez, P. M. (2016). Las competencias de los docentes para el uso y manejo de las TIC en la implementación del Proyecto Educativo Canaima. Caso: Escuela Municipal San José. Acción Pedagógica, 25, 22-32.

Vega, P. G., \& Vasquez, C. Y. (2021). Acompañamiento pedagógico y desempeño docente en educación primaria. Espacios, 42(21), 1-10. https://doi.org/10.48082/espacios-a21v42n19p01

Villegas, M., Gonzales, S., Gonzales, F., Pichardo, G., \& Rodriguez, I. (2017). 
Conocimientos previos sobre acompañamiento pedagógico. Ciencia y Sociedad, 42(1), 89-103. https://revistas.intec.edu.do/index.php/ciso/article/view/1065

Vygotsky, L. (1995). Pensamiento y lenguaje (Fausto (ed.)). https://play.google.com/books/reader?id=mCJCYufSHQC\&pg=GBS.PP1\&hl=es_419

Vygotsky, L. (2006). Psicología del arte (Paidós (ed.); 1 st ed.). https://play.google.com/books/reader?id=xm-W7HJnQkC\&pg=GBS.PP8.w.0.2.52_16\&hl=es_419

Zárate, D. (2021). Acompañamiento pedagógico directivo en el desempeño docente en la institución educativa 3023 Pedro Paulet Mostajo San Martin de Porras 2021 [Universidad César Vallejo]. http://repositorio.uncp.edu.pe/bitstream/handle/UNCP/3000/Silva Acosta.pdf? sequence $=1 \&$ isAllowed $=y \% 0$ Ahttps://repositorio.comillas.edu/xmlu i/handle/11531/1046 